\title{
Acupuncture Point
}

National Cancer Institute

\section{Source}

National Cancer Institute. Acupuncture Point. NCI Thesaurus. Code C93246.

An anatomic point, as defined by the Standard Acupuncture Nomenclature, that can be stimulated via various techniques, including the insertion and withdrawal of needles, the application of heat, massage, laser, electrical pulsation, or a combination of these methods. 